\title{
Diseño e implementación de un sistema prototipo de encendido y transferencia inalámbrica de datos de un espectrómetro para la detección de derrames de petróleo
}

\author{
Alexander Cárdenas \\ Escuela Politécnica Nacional. Facultad de Ingeniería Eléctrica y Electrónica \\ alexander.cardenas@epn.edu.ec \\ Eduardo Ávalos \\ Escuela Politécnica Nacional. Facultad de Ingeniería Eléctrica y Electrónica \\ eduardo.avalos@epn.edu.ec \\ Rolando Sáenz* \\ Escuela Politécnica Nacional. Facultad de Ingeniería Eléctrica y Electrónica \\ rolando.saenz@epn.edu.ec
}

Recibido: 01 de septiembre / Aprobado: 30 de noviembre 2017

\section{Resumen}

Este documento presenta el diseño, implementación y pruebas de un sistema prototipo de detección de derrames de petróleo basado en la utilización de un sensor de resistencia eléctrica de suelo, este prototipo enciende un espectrómetro del cual se toman datos que posteriormente son transmitidos inalámbricamente para determinar si existe o no contaminación generada por un derrame de petróleo. El prototipo se encuentra divido en 2 subsistemas: subsistema sensor-Arduino-actuador y subsistema de transferencia inalámbrica de datos. El subsistema sensor-Arduino-actuador es el encargado de detectar un cambio en la resistencia eléctrica del suelo y encender el espectrómetro, mientras que el subsistema de transferencia inalámbrica de datos se encarga de tomar los datos 
del espectrómetro y enviar los mismos como un mensaje de correo electrónico utilizando una conexión inalámbrica con una red de datos celular. El subsistema de transferencia inalámbrica de datos se encuentra implementado mediante una tarjeta Raspberry Pi y un módulo GSM/GPRS. Todos los programas utilizados para controlar tanto la placa Arduino como la placa Raspberry Pi se encuentran implementados con software libre.

Palabras clave: Arduino, espectrometro, Raspberry Pi.

\section{Abstract}

This paper presents the design, implementation and testing of a prototype of an oil spill detection system based on the use of an electric soil resistance sensor, this prototype turn on a spectrometer from which data is then taken wirelessly to determine if there is or is not pollution generated by an oil spill. The prototype is divided into 2 subsystems which are: Sensor-Arduino-Actuator subsystem and wireless data transfer subsystem. The Sensor-Arduino-Actuator subsystem is responsible for detecting a change in the electrical resistance of the ground and turning on the spectrometer, while the wireless data transfer subsystem is responsible for taking the spectrometer data and sending the same as a message of email using a wireless connection to a cellular data network. The wireless data transfer subsystem is implemented using a Raspberry Pi card and a GSM / GPRS module. All programs used to control both the Arduino board and the Raspberry Pi board are implemented with free software.

Keywords: Arduino, espectrómeter, Raspberry Pi. 


\section{Introducción}

$\mathrm{L}$ os derrames de petróleo son causa de grandes pérdidas económicas tanto para empresas petroleras como para actividades agrícolas locales, pero sin duda alguna los derrames de petróleo son una de las causas de mayor contaminación ambiental, los efectos que generan son de acción inmediata y duradera en el tiempo, muchos de estos efectos llegan inclusive a ser irreversibles. El presente proyecto se basa en el uso de sensores, microcontroladores y comunicaciones inalámbricas con el fin de generar una respuesta temprana ante una posible situación de derrame de petróleo.

El uso de microcontroladores $\mathrm{p}$ ara la automatización de procesos es una práctica ampliamente difundida, pues estos son capaces que de realizar operaciones previamente grabadas en su memoria. Estos dispositivos son capaces de trabajar en conjunto con sensores y actuadores. Los sensores permiten obtener datos sobre el entorno, estos datos son enviados hacia los microcontroladores para que sean procesados y acorde a estos se tomen o no acciones, mientras que los actuadores son dispositivos que tras recibir una señal de un microcontrolador realizan una acción en particular.

En la actualidad para realizar análisis de muestras de petróleo se utilizan espectrómetros, los espectrómetros aprovechan las características de fluorescencia de algunos de los hidrocarburos presentes en el petróleo para realizar sus mediciones, esta técnica se denomina LIF (Fluorescencia Inducida por Láser)[1].

Cuando la luz interactúa con la materia se pueden generar varios procesos tales como: reflexión, dispersión, absorbancia, fluorescencia, fosforescencia, etc.

La fluorescencia es un fenómeno que presentan algunas sustancias, se basa en que estas sustancias son capaces de absorber luz, generalmente en el rango del ultravioleta, para luego emitir luz con una longitud de onda mayor. La energía emitida por la sustancia en forma de luz es menor que la energía que fue absorbida pues parte de esta energía es emitida en forma de calor. Este fenómeno es tan breve que ocurre en el orden de los nanosegundos, es tan rápido que tan pronto como existe una excitación de la sustancia el fenómeno aparece y tan pronto como desaparece la excitación el fenómeno de igual manera desaparece [2].

En el presente proyecto se pretende crear un prototipo que ante una posible situación de derrame de petróleo, basándose en un incremento en la resistencia eléctrica del suelo, encienda un espectrómetro y envíe inalámbricamente los datos recolectados por este equipo para su análisis y determinar así si existe o no la presencia de petróleo. 


\section{Metodología}

Los sensores son dispositivos con la capacidad de imitar la forma en la que las personas perciben el entorno. Su uso se encuentra tan extendido que actualmente todo dispositivo electrónico presenta algún tipo de sensor. Estos dispositivos son capaces de obtener información del entorno y transmitirla. Esta información puede ser procesada para generar respuestas o iniciar procesos [3].

Arduino es una plataforma de desarrollo de prototipos de hardware y software libre, está compuesta principalmente de una placa con un microcontrolador programable. Los microcontroladores que utiliza Arduino son del tipo AVR, que es una familia de microcontroladores fabricada por la marca Atmel. Las placas Arduino presentan un número variable de pines, dependen del modelo de Arduino, los que se encuentran conectados a las entradas y salidas del microcontrolador. Los pines pueden ser entradas y salidas digitales así como entradas analógicas, las cuales permiten conectar e interactuar con facilidad con sensores y actuadores.

Los microcontroladores son circuitos integrados que tienen la característica de ser programables. Estos dispositivos son capaces de realizar tareas programadas previamente de manera autónoma, disponen de una unidad central de procesamiento (CPU), esta unidad es la encargada de realizar todas las tareas programadas y controlar que estas se ejecuten correctamente.

Los microcontroladores presentan una serie de entradas y salidas, estas pueden ser digitales o analógicas. Las entradas o salidas permiten conectar los microcontroladores con una gran cantidad de periféricos, las entradas analógicas están a su vez conectadas a un conversor analógico-digital (CAD), que tiene como función tomar una señal analógica y transformarla a un valor binario, es decir transforman señales analógicas en señales digitales. En general los microcontroladores que usa Arduino poseen un CAD de 10 bits.

La conversión analógica digital es un proceso que empieza cuando la variable física entra al sistema, una vez en el sistema esta señal analógica (continua en tiempo y en amplitud) es muestreada. El proceso de muestreo, también conocido como sampling, consiste en tomar muestras de la señal analógica en determinados instantes de tiempo con lo cual se obtiene una señal equivalente a la analógica pero discreta en tiempo. Los instantes de tiempo en los que se muestrea la señal obedecen al teorema del muestreo que establece que una señal debe ser muestreada a una frecuencia de al menos dos veces la frecuencia más alta contenida en la señal.

Tras el muestreo de la señal esta pasa a ser cuantificada con lo que se obtiene una senal discreta tanto en tiempo como en amplitud. En la cuantificación se aproxima cada una de las muestras a un único valor del intervalo de cuantificación en el que caen. 
Finalmente se realiza el proceso de codificación, éste consiste en asignar una palabra binaria a cada intervalo de cuantificación

Los actuadores son dispositivos que transforman algún tipo de energía para generar una fuerza que ejerce un cambio de posición, velocidad o estado de un elemento mecánico. Los actuadores reciben órdenes de los microcontroladores y generan una salida para activar un elemento final que es parte de un proceso automatizado.

Raspberry Pi son una serie de micro computadores con una alta capacidad de procesamiento, incorporan todas sus partes en una placa de circuito impreso (PCB) de un tamaño no mucho mayor al de una tarjeta de crédito. Estos microcomputadores son compatibles con una gran cantidad de periféricos, estos incluyen: teclados, ratones, pantallas LCD, impresoras, cámaras, etc. Dichos micro computadores utilizan como disco duro tarjetas SD o micro SD donde alojan el sistema operativo.

Todas las versiones de Raspberry existentes cuentan con puertos HDMI y USB así como con una interfaz denominada GPIO (Entradas/Salidas de Propósito General). Además de las interfaces anteriores, todas las versiones de placas Raspberry incluyen también un puerto Ethernet. Los procesadores utilizados en las placas Raspberry son de arquitectura ARM, lo cual les permite ejecutar operaciones complejas con un bajo consumo de energía. Debido a su alta capacidad de procesamiento estos dispositivos pueden utilizar algunos sistemas operativos Linux [5].

La interfaz GPIO son un conjunto de pines existentes en todas las placas Raspberry, según el modelo pueden estos contener 26 o 40 pines. Estos pines son entradas y salidas digitales y permiten a las placas Raspberry interactuar con el mundo exterior. En estos pines se pueden conectar sensores o recibir señales provenientes de computadores u otros dispositivos. Todos los pines GPIO pueden configurarse mediante el sistema operativo para cumplir funciones de entradas o salidas digitales y así automatizar procesos.

Raspbian es un sistema operativo optimizado -una de las tantas distribuciones gratuitas existentes de Linux- para trabajar con el hardware de las placas Raspberry Pi. Raspbian está basado en Debian (otro sistema operativo también basado en Linux). Un sistema operativo es un conjunto básico de programas y utilidades que permiten que Raspberry funcione.

Los módulos GSM/GPRS son dispositivos inalámbricos capaces de conectarse a las redes de telefonía celular tal cual lo haría un teléfono celular y utilizar todos los servicios que estas provean, como llamadas de voz, mensajes (SMS), email, internet, etc. Estos dispositivos son de un tamaño reducido y contienen todo el hardware necesario para usar las redes de telefonía celular, pueden ser controlados fácilmente por microcontroladores o computadores debido a que presentan interfaces de comunicación serial. 
La rápida evolución de la telefonía celular ha permitido que los sistemas celulares presenten nuevas y mejores características que se adapten a las cambiantes necesidades de las personas. En sus inicios los sistemas celulares eran analógicos y estrictamente para llamadas de voz, posteriormente aparecieron servicios como mensajes de texto cortos (SMS) y conexiones a internet, todo esto gracias a que los sistemas pasaron a ser digitales y las velocidades de transmisión de datos se fueron incrementando rápidamente.

GSM es una tecnología celular de segunda generación, es la tecnología celular con más suscriptores en el mundo, presenta ya un servicio de voz digital y con velocidades de transmisión de datos de hasta $14.4 \mathrm{kbps}$. GSM fue la primera tecnología en introducir el uso de tarjetas SIM así como implementar el servicio de mensajes cortos (SMS). GSM como tal no permite a los usuarios conectarse a internet, sin embargo su infraestructura es fundamental para lograrlo [6].

La arquitectura de GSM se subdivide en tres subsistemas:

- Estación Móvil: está conformado por el dispositivo terminal de usuario y la tarjeta SIM. El dispositivo terminal de usuario puede ser un teléfono celular, una tablet, una laptop o inclusive un módem.

- Subsistema de Estación Base: también denominado "red de radio", es el encargado de gestionar la interfaz de radio, contiene todos los nodos y funcionalidades que son necesarios para conectar de forma inalámbrica a los suscriptores móviles a través de la interfaz de radio a la red.

- Subsistema de Red: denominada "núcleo de la red". Cumple funciones relacionadas con la conmutación de llamadas, para la gestión de abonados y la gestión de la movilidad. La tarea más importante de este subsistema es el establecimiento, control y enrutamiento de llamadas entre diferentes centros de conmutación fijos y móviles y otras redes.

GPRS es una tecnología 2.5G, considerada una extensión de GSM debido a que introduce ciertos componentes y actualiza el software de la infraestructura propia de GSM para poder funcionar, con lo cual los operadores de telefonía celular no necesitan reemplazar la infraestructura ya existente.

GPRS permitió por primera vez a los dispositivos móviles conectarse a internet a través de una infraestructura de conmutación de paquetes, además permitió usar el servicio de correo electrónico así como el servicio de mensajes multimedia (MMS). [8]

GPRS trabaja con velocidades de datos superiores a las de GSM, estas pueden llegar a ser teóricamente de 80 kbps en el downlink y de 40 kbps en el uplink, aunque en la práctica llegan a ser mucho menores [6]. 


\section{Diseńo e implementacion del sistema}

En esta sección se muestra el diseño e implementación del sistema prototipo propuesto, que está basado en la utilización de plataformas de hardware y software libre.

\section{- Subsistema de transferencia inalámbrica de datos}

Los datos generados por el espectrómetro son pares longitud de onda - intensidad fotónica, estos datos son generados en un archivo con extensión .txt. El tamaño del archivo generado, cada vez que se enciende el espectrómetro, es de 57 kilobyte. Estos archivos generados serán enviados por medio de correo electrónico, a través de una cuenta creada solo para este propósito.

Debido a que los datos serán enviados mediante correo electrónico se necesita de una tecnología celular que cuente con una red datos para tener una conexión a internet. Entre las tecnologías existentes en el país con estas características se encuentran: GPRS, HSPA+, LTE y LTE A. La tecnología GPRS es la más antigua de todas las mencionadas y con la velocidad de transmisión de datos más baja, sin embargo para el proyecto actual, estas velocidades son suficientes para un correcto funcionamiento debido a que no se necesita un gran ancho de banda para usar el servicio de correo electrónico.

El principal factor para determinar si la solución en base a la tecnología GPRS es factible, es la cobertura. Desde este aspecto la cobertura que presenta esta tecnología celular es la mejor con respecto a las demás tecnologías.

El módulo utilizado en este proyecto es un módulo SIM800L EVB. Este módulo permite conectar el prototipo con la red de datos celular, es un módulo de tamaño reducido que opera a $5 \mathrm{~V}$ y posee un slot para tarjetas $\mu$ SIM. Es un módulo de cuádruple banda que trabaja a las frecuencias de GSM $850 \mathrm{Mhz}$, EGSM $900 \mathrm{MHz}$, DCS $1800 \mathrm{MHz}$ y PCS 1900 Mhz. La velocidad máxima teórica que se puede tener con este módulo es de 85,6 kbps en el downlink.

Para extraer y enviar los datos del espectrómetro y controlar el funcionamiento del módulo GSM/GPRS se utilizó una placa Raspberry Pi 2. Esta placa es un microcomputador que consta de un procesador quad-core ARM Cortex que opera a $900 \mathrm{Mhz}$ y un $1 \mathrm{~Gb}$ de memoria RAM como características principales. Además cuenta con: 4 puertos USB, 40 pines entrada/salida de propósito general (GPIO), un puerto HDMI, un puerto Ethernet, una ranura para tarjetas microSD, entre otras interfaces que para el presente proyecto no son utilizadas.

El microespectrómetro utilizado es de la compañía Ocean Optics, cuenta con un kit de desarrolladores enfocado a la implementación de proyectos de investigación. Este kit se encuentra implementado con placas Raspberry pi 2 y usan como sistema operativo una distribución de Linux modificada. Este sistema operativo cuenta con las mismas características que la versión original de Raspbian Wheezy 
pero además cuenta con librerías y controladores propios del microespectrómetro previamente instalados.

Estos microespectrómetros no cuentan con una fuente de luz propia, razón por la cual para realizar las pruebas de funcionamiento del equipo se implementó un espectrómetro usando el microespectrómetro y una fuente de luz de $390 \mathrm{~nm}$.

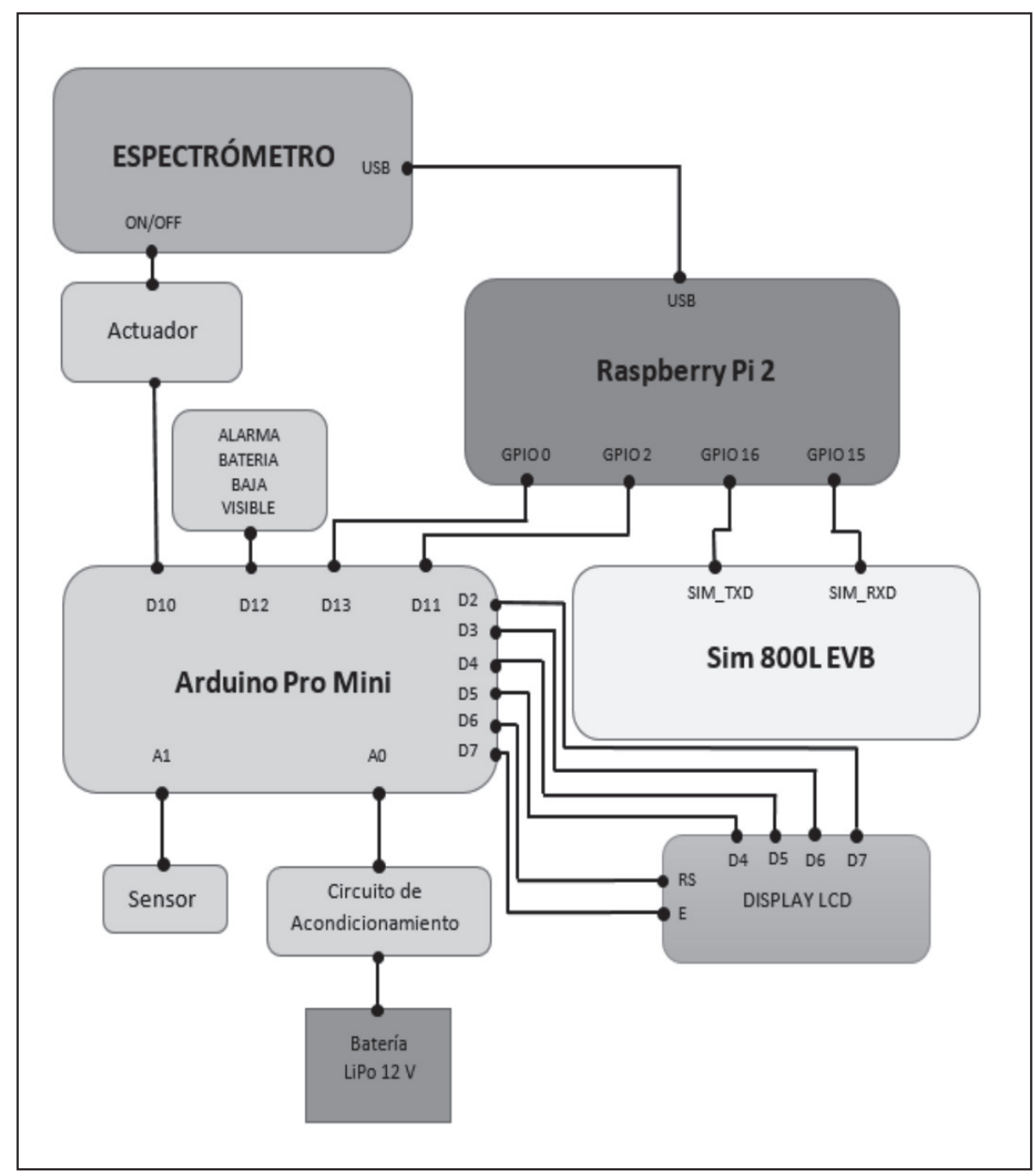

Figura 1. Diagrama de conexión de dispositivos.

\section{- Subsistema sensor-Arduino-actuador}

El sensor utilizado basa su funcionamiento en la resistencia eléctrica que presente el suelo, el sensor está conformado de una placa electrónica y una sonda, este sensor cuenta con dos salidas, una salida digital y una salida analógica. 
La salida digital para este proyecto no es utilizada, mientras que la salida analógica envía hacia la placa Arduino un voltaje comprendido entre $0 \mathrm{~V}$ y $5 \mathrm{~V}$ equivalente a la resistencia eléctrica presente en la sonda insertada en el suelo.

La sonda del sensor se muestra en la figura 2. La resistencia eléctrica del suelo que se mide es aquella que se encuentra entre las paredes del tubo de cobre y la punta que atraviesa el interior del tubo, del mismo material.

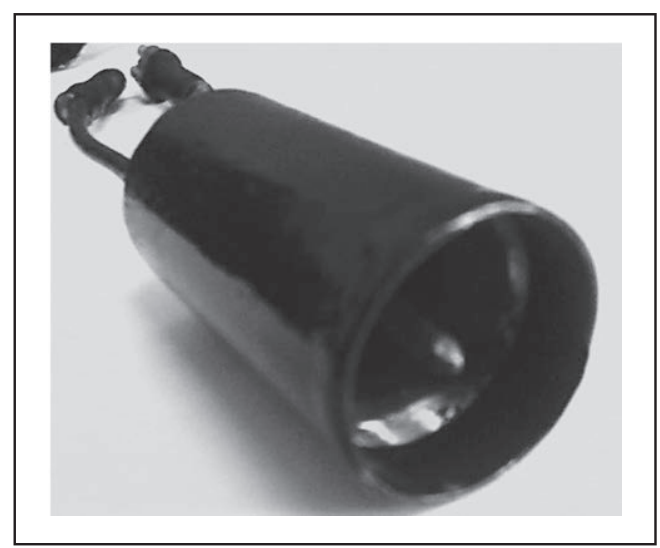

Figura 2. Sonda del sensor de resistencia eléctrica de suelo.

Para el proyecto se utilizó una placa de desarrollo Arduino Pro-Mini. Esta placa cuenta con un microcontrolador Atmel Atmega 328, 14 pines entrada/salida digitales y 6 entradas analógicas, cada una está conectada a un conversor analógico digital de 10 bits [7].

Existen dos versiones: una a $3.3 \mathrm{~V}$ y $8 \mathrm{Mhz}$ y otra a $5 \mathrm{~V}$ y $16 \mathrm{Mhz}$, para este proyecto se escogió la versión de $5 \mathrm{~V}$ y $16 \mathrm{Mhz}$, esto debido a que tanto el módulo GPRS y la tarjeta Raspberry pi 2 usados para la transmisión inalámbrica de datos operan de igual manera con $5 \mathrm{~V}$, evitando el uso de circuitería adicional para ajustar el voltaje.

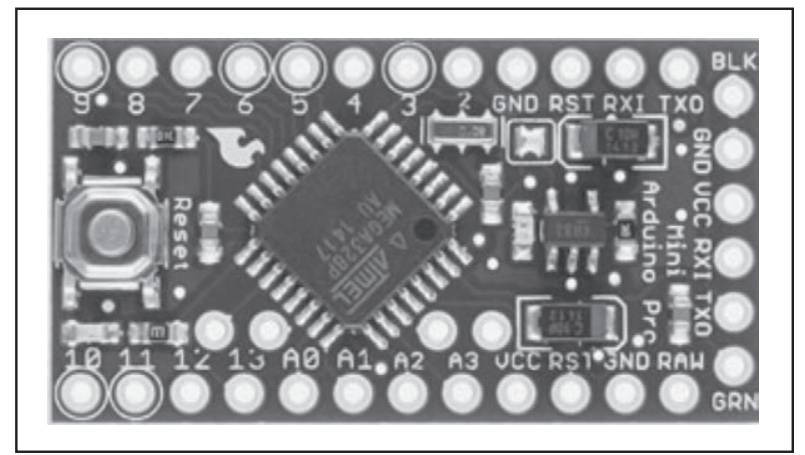

Figura 3. Arduino Pro Mini. 
El actuador que se implementó para este proyecto es un conjunto de elementos que está conformado por un relé electromecánico, un transistor, un diodo y una resistencia. La figura 4 muestra el circuito esquemático del actuador.

Para la alimentación de todo el prototipo se hizo uso de una batería LiPo de 3 celdas, junto con un regulador de voltaje variable StepDown. Este regulador fue configurado para entregar $5 \mathrm{~V}$, voltaje con el cual operan todos los dispositivos del prototipo.

La batería utilizada en este proyecto es una batería Li-Po de 3 celdas con un voltaje nominal de $11.1 \mathrm{~V}$ y $5000 \mathrm{mAh}$. El voltaje de la batería puede llegar a ser de hasta $12,6 \mathrm{~V}$ cuando está totalmente cargada.

\section{Pruebas y resultados}

Para determinar el comportamiento del suelo ante la presencia de petróleo y la manera en la que responde el sensor, se implementó un sistema de adquisición de datos utilizando la misma placa Arduino Pro-Mini y el sensor del prototipo.

Para implementar este sistema de adquisición de datos se hizo uso del programa PLX-DAQ que permite recolectar los datos obtenidos por la placa Arduino en una hoja de cálculo de Excel en tiempo real. En las hojas de cálculo se almacenaron los valores obtenidos por el sensor así como la hora en la que la medición fue realizada y el tiempo transcurrido entre cada una de las mediciones

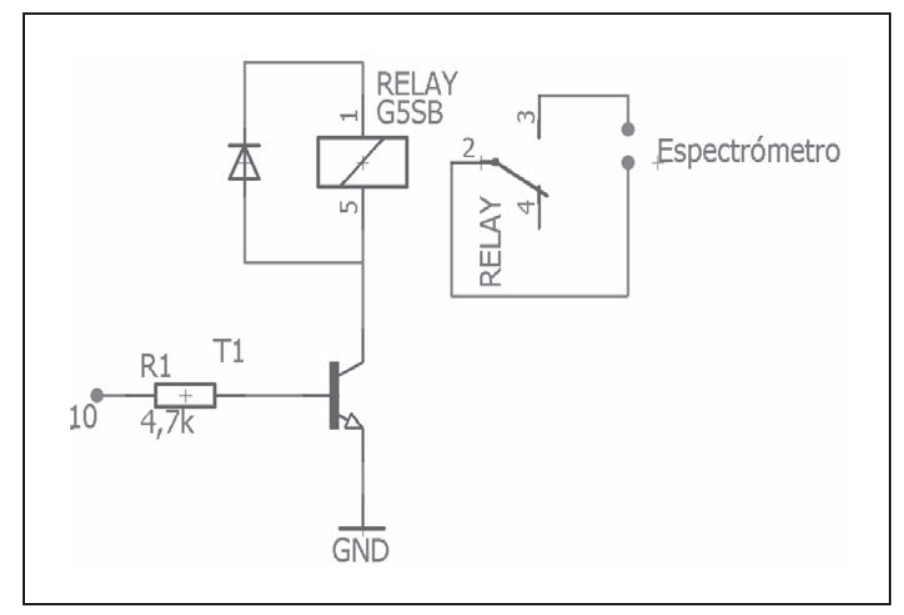

Figura 4. Diseño esquemático del actuador.

La figura 5 muestra una gráfica con los datos obtenidos al contaminar suelo humífero con petróleo a distintos niveles de humedad.

Los puntos rojos que se observan en la gráfica indican el momento en el que se realizó la contaminación con petróleo.

La figura 6 muestra una gráfica con los datos obtenidos al contaminar suelo arenoso con petróleo a distintos niveles de humedad. 
La figura 7 muestra una gráfica con los datos obtenidos al contaminar suelo arcilloso con petróleo a distintos niveles de humedad.

El equipo fue colocado en una caja metálica, en la cual un botón de encendido general fue ubicado en la parte superior, mientras que a los costados se realizaron orificios para colocar la antena del módulo GSM/GPRS y dar una salida a los cables de conexión con el espectrómetro y la sonda. El equipo ensamblado se muestra en la figura 8.

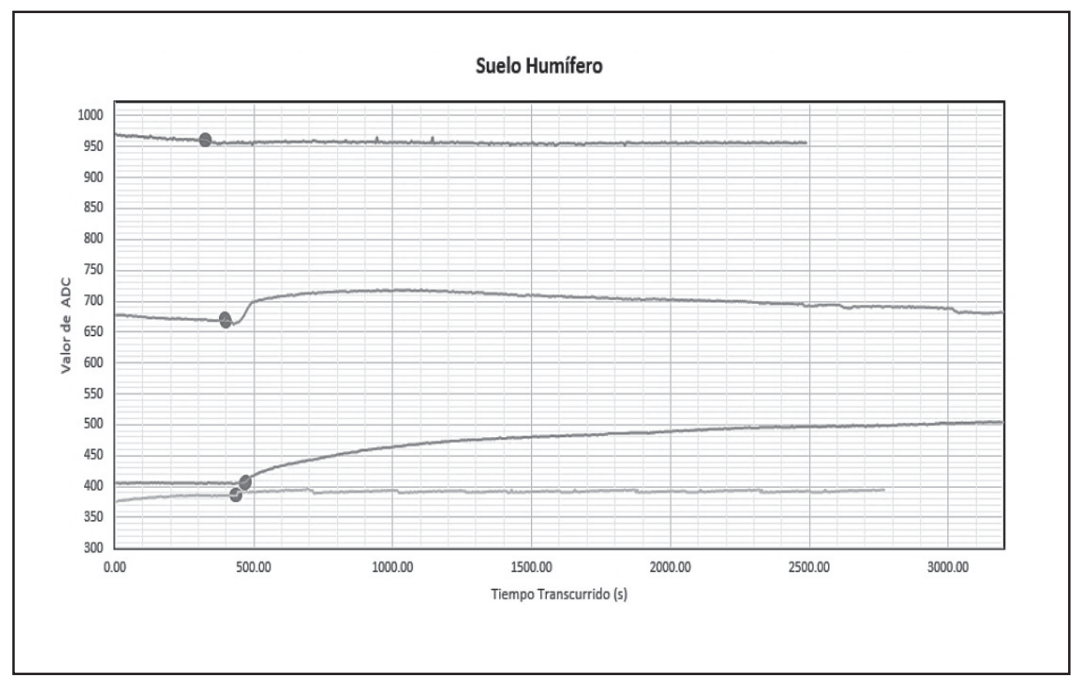

Figura 5. Comportamiento del suelo humífero ante la presencia de petróleo.

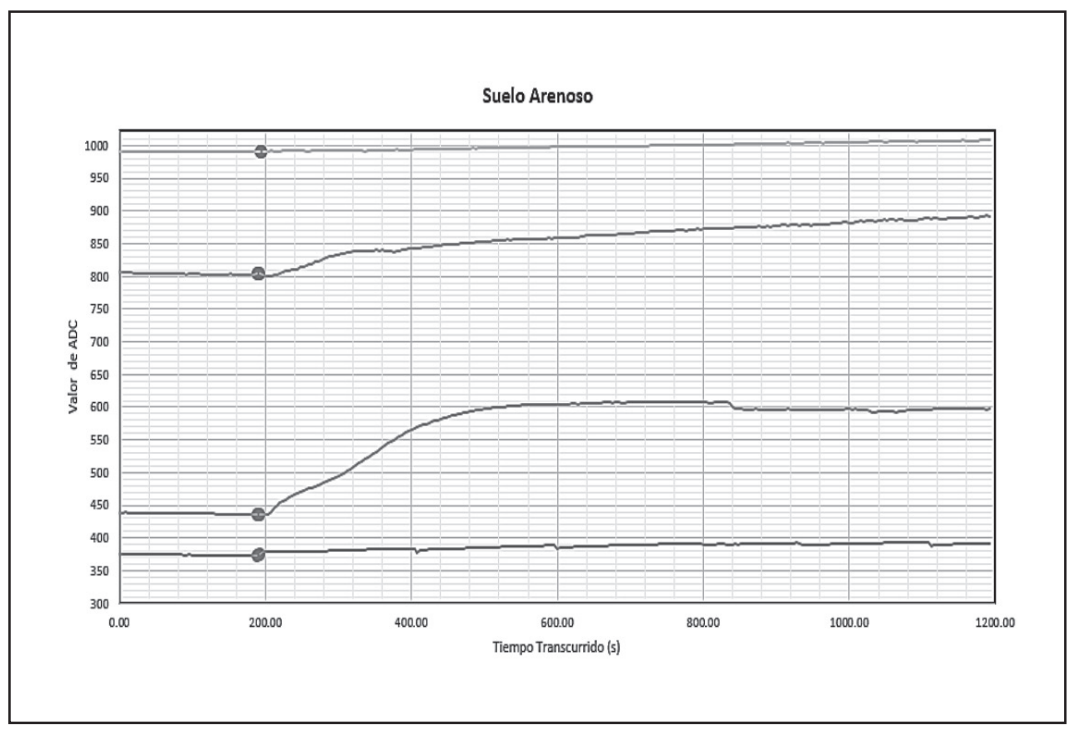

Figura 6. Comportamiento del suelo arenoso ante la presencia de petróleo. 


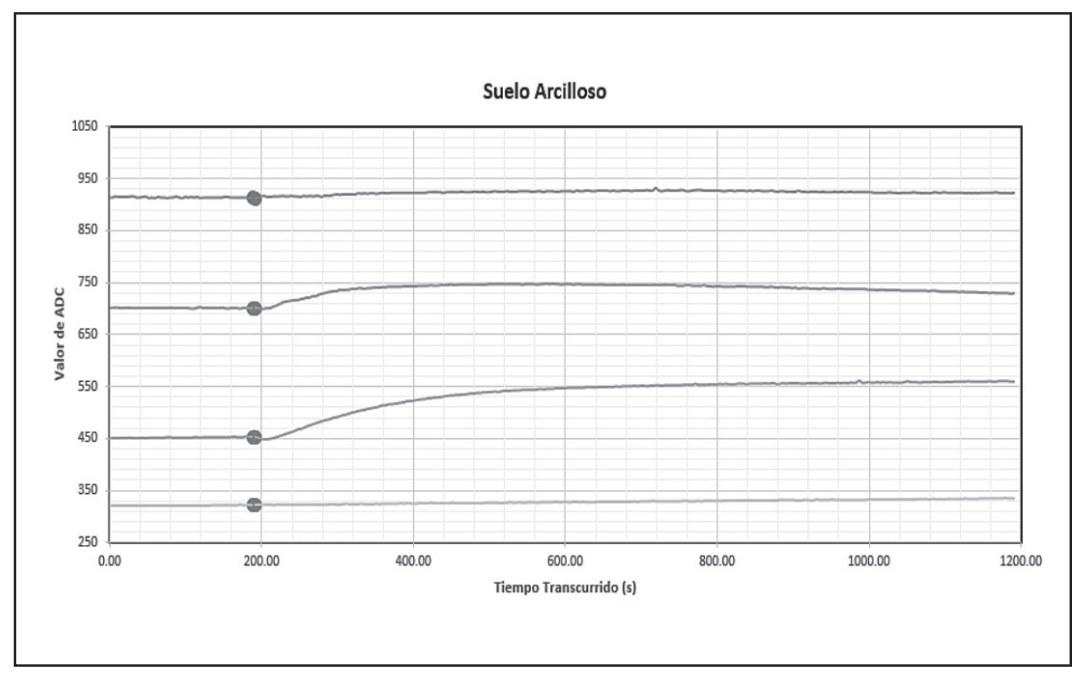

Figura 7. Comportamiento del suelo arcilloso ante la presencia de petróleo.

Para graficar los datos obtenidos del espectrómetro tras ser enviados por mensajes de correo electrónico, se desarrolló un software en Matlab. Los resultados de suelo contaminado con petróleo se muestran en la figura 10 .

Como se puede observar, existe un pico alrededor de los $510 \mathrm{~nm}$ perteneciente a un fenómeno de fluorescencia producido al iluminar suelo contaminado con una fuente de luz de $390 \mathrm{~nm}$.

Algo distinto sucede cuando se envían datos de suelo sin contaminar con petróleo, en este caso el pico antes mencionado es inexistente.

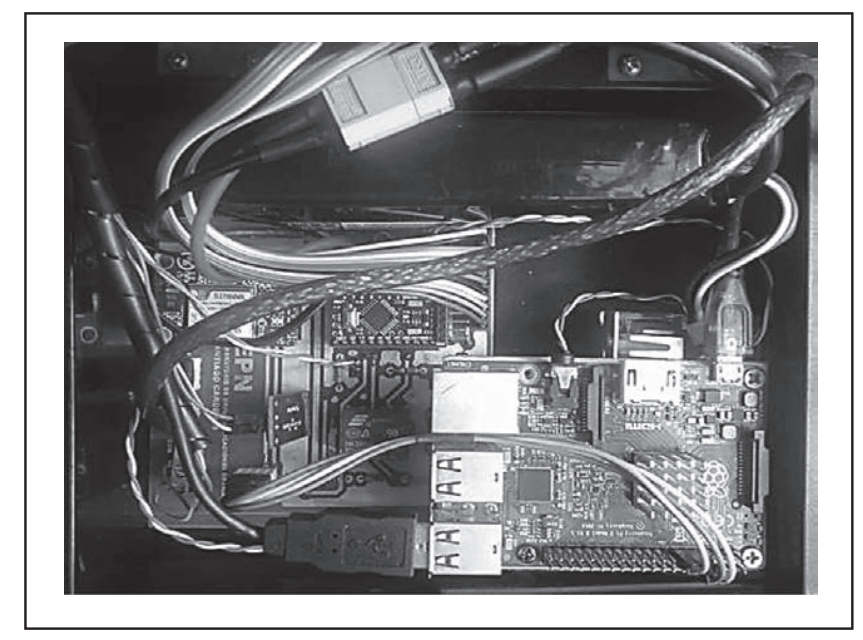

Figura 8. Vista interna del equipo prototipo ensamblado. 
La figura 9 muestra una vista externa del equipo prototipo.

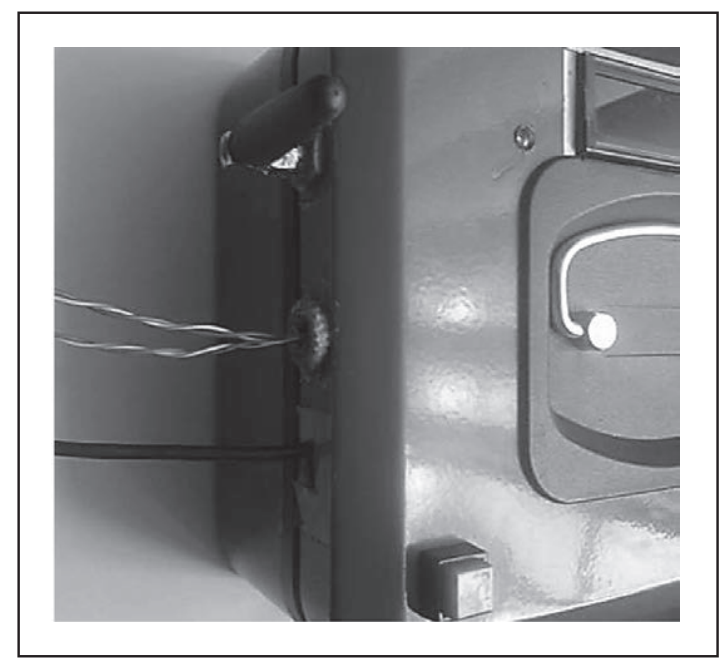

Figura 9. Vista externa del equipo prototipo.

Los picos más grandes observados alrededor de los $390 \mathrm{~nm}$ corresponden a un fenómeno de reflexión debido a la fuente de luz utilizada en el proyecto.

En la figura 12 se puede observar el equipo completamente instalado.

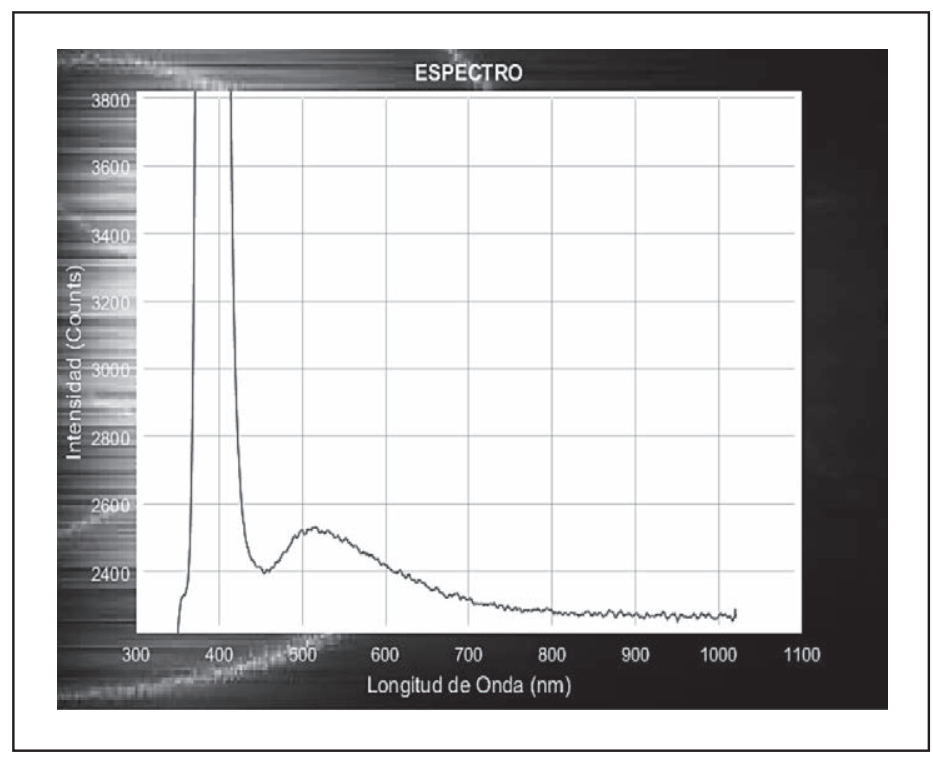

Figura 10. Vista externa del equipo prototipo.

La figura 11 muestra la gráfica obtenida de suelo sin contaminar. 


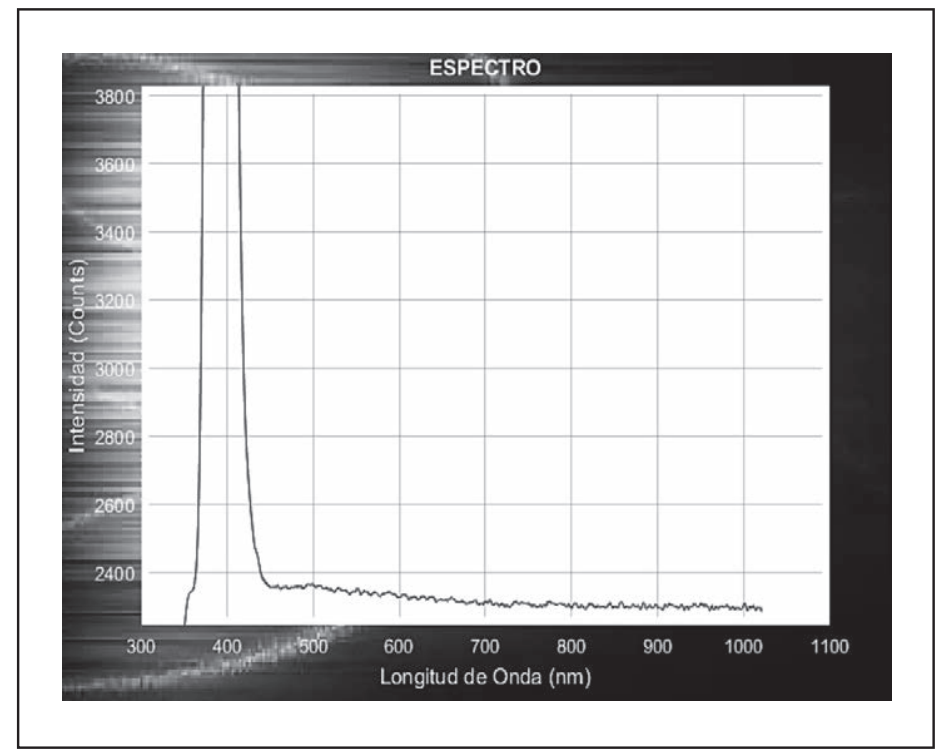

Figura 11. Vista externa del equipo prototipo.

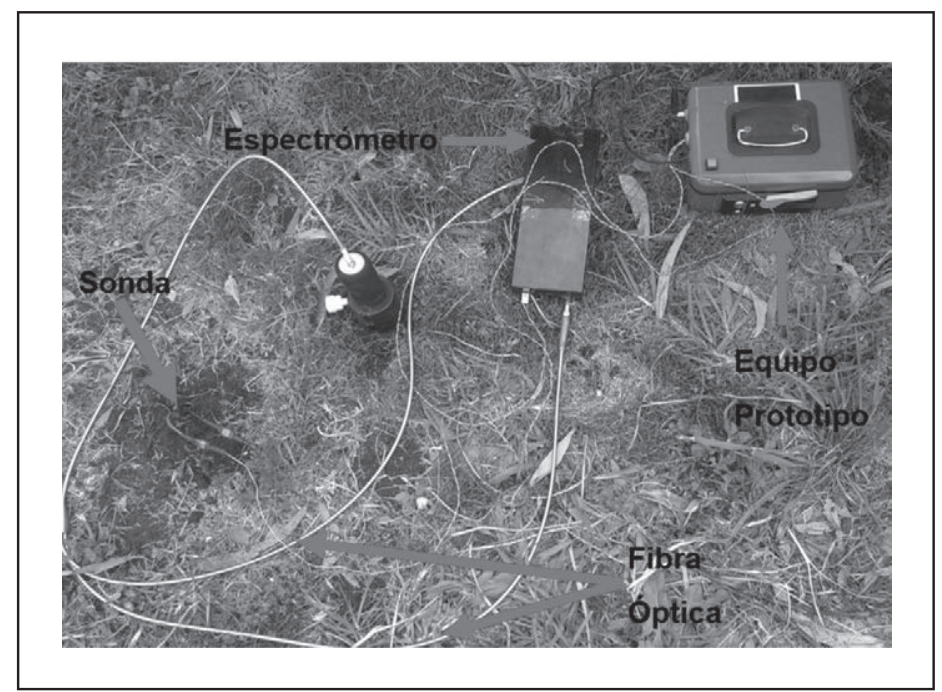

Figura 12. Equipo prototipo instalado.

\section{Conclusiones}

El equipo prototipo permite determinar si existe o no un derrame de petróleo mediante el análisis de los datos enviados, los espectros recibidos muestran un claro pico en los $510 \mathrm{~nm}$ correspondiente a un fenómeno de fluorescencia cuando existe la presencia de petróleo, mientras que la presencia de este pico es inexistente en suelo que no se encuentra contaminado con petróleo. 
Existen varios factores ambientales como temperatura, humedad, radiación solar que generan un incremento en la resistencia eléctrica del suelo, de manera similar al que genera el petróleo en contacto con el suelo, lo que hace que el equipo prototipo encienda el espectrómetro y se envíen los datos a pesar de que no exista una contaminación con petróleo.

La tecnología celular GPRS es adecuada para transmitir la pequeña cantidad de datos generados por el espectrómetro, cada vez que éste sea encendido por una posible situación de derrame de petróleo, a pesar de no ser la tecnología más actual disponible en el mercado ni la más rápida. Sin embargo GPRS es la tecnología más adecuada para transmitir los datos generados desde el punto de vista de cobertura puesto que esta tecnología usa la cobertura de GSM, que es en este aspecto la tecnología celular que mejores prestaciones brinda.

\section{Referencias}

[1] UNESCO. Determinación de los hidrocarburos del petróleo en los sedimentos, 1982. [En línea]. Available: http://unesdoc.unesco.org/images/0005/000526/ 052655so.pdf. [Último acceso: febrero 2017].

[2] J. Lakowick. Principles offlourescence spectroscopy. Nueva York: Springer Science + Business Media, 2004.

[3] A. Serna, F. Ros y J. Rico. Guía práctica de sensores. España: Creaciones Copyright, S.L., 2010.

[4] O. Torrente. Arduino. Curso práctico de formación. México: Alfaomega Grupo Editor, 2013.

[5] Raspberry PI Foundation. GPIO: Raspberry pi models $A$ and B. [En línea]. Available: https://www.raspberrypi.org/documentation/usage/gpio/. [Último acceso: febrero 2017].

[6] M. Sauter. From GSM to LTE: an introduction to mobile networks and mobile broadband. Alemania: John Wiley and Sons, 2011.

[7] Arduino, «Arduino Pro Mini», 2017. [En línea]. Available: https://www.arduino.cc/en/Main/ArduinoBoardProMini. [Último acceso: febrero 2017]. 